A Colour Atlas of Ocular Tumours

By M. A. Bedford. Pp. 78, illustrated. Wolfe Medical

Publications, London, 1979. $£ 12.00$.

The Wolfe Medical Publications Atlases are well known and the Colour Atlas of Ocular Tumours is a useful but specialized new addition to the series. The page size $(260 \mathrm{~mm} \times 190$ $\mathrm{mm}$ ) larger than most previous Wolfe Atlases complements this particular subject very well. A strong shiny cloth binding is in keeping with the high standard of production of the Wolfe Atlas series.

Michael Bedford is an expert in ocular tumours and it is fitting that his extensive knowledge of this ophthalmological sub-speciality should be summarized in this Atlas. The 224 colour photographs amply cover the clinical appearance of most ocular and eyelid tumours, with a very brief descriptive text for each photograph. A number of pages have a rather crowded layout where the text of one photograph appears to run into the text of an adjoining one.

$A$ Colour Atlas of Ocular Tumours will be a very useful reference and read for all ophthalmic surgeons in training and might also prove useful for specialized oncologists and radiotherapists to see their specimens well illustrated in situ. The absence of a detailed text and histology of course reduces the effectiveness of the Atlas for more experienced ophthalmic surgeons which is a pity as the excellent photographs deserve a wide readership.

However, it is well understood that the Wolfe Atlas series do not claim to be comprehensive publications on their subjects and a Colour Atlas of Ocular Tumours may be considered a worthy easy-to-read addition to the Wolfe Atlas series.

\section{A Colour Atlas of Peripheral Vascular Diseases.}

By W. F. Walker. Pp. 112, illustrated. Wolfe Medical Publications, London, 1980. £15.00.

Vascular disease in its various forms accounts for nearly one half of the deaths in our so-called civilized world. Peripheral arterial diseases are responsible for much suffering and loss of work, as well as being the indication for the great majority of amputations today. Varicose veins and their complications are the most frequent cause of referral to general surgical out-patients and, indeed, most hospitals have to set up special clinics to deal with these patients. There is therefore a wealth of important pathological and clinical conditions which are of intense interest to today's medical student, and practitioners. Mr William Walker, who is Consultant Surgeon at Ninewells Hospital, Dundee, has produced a magnificent colour atlas of both common and unusual conditions of the arteries, veins and lymphatic system. His material ranges over acute and chronic ischaemia, gangrene, cerebrovascular disease, aneurysms, blood vessel tumours, trauma, varicose veins, deep vein thrombosis and its consequences, lymphoedema and tumours of the lymphatic vessels, illustrating their pathology, clinical features and angiography. Like the other volumes in this well known series of medical atlases, this serves as a magnificent supplement to the standard textbooks on vascular surgery. Medical students and postgraduate surgical trainees will benefit greatly from a careful study of this atlas - the next best thing to seeing the patients themselves.

\section{A Colour Atlas of Tumour Histopathology.}

By N. F. C. Gowing. Pp. 272, illustrated. Wolfe Medical Publications, London, 1980. £35.00.

This Atlas by one of this country's foremost tumour histopathologists is a very welcome addition to the series. Being directed primarily at trainee pathologists it is arranged by site, and each organ or system has a section devoted to it. Most tumours, both common and rare, arising in each site are illustrated, mainly by photomicrographs of $\mathbf{H} \&$ E-stained sections. Where helpful, appropriate special stains, photo-ฏ graphs or gross specimens and occasional electron micro- $\frac{2}{Z}$ graphs are illustrated. A number of proliferative lesions $\frac{\mathbb{Q}}{\infty}$ which may be mistaken for malignant tumours are also 2 included.

As the title implies, this is purely an atlas and the text is confined to clear descriptive captions to each photograph, many of which include brief clinical details or points of differential diagnosis.

Professor Gowing has clearly drawn from his vast collec- $\frac{\bar{\sigma}}{\overline{\frac{D}{0}}}$ tion of material to produce this book and is to be congratu- $\frac{\sigma}{-}$ lated on its comprehensiveness and the very high quality of $\mathbb{Q}$ the illustrations both at low and high magnifications.

This volume will provide a useful adjunct to the standard text books for the trainee pathologist but will also be of $\vec{\nabla}$ interest to all who deal with tumours, both clinicians and? pathologists. It has already been helpful in solving one or two $\overrightarrow{\vec{c}}$ tricky slide seminar problems!

It is a pity that its price may deter those for whom it is primarily intended from buying their own copy but it will be a을 most useful addition to the book shelf of every histopathology 3 . laboratory.

The Development of the Infant and Young Child. Normal and $\dot{\sigma}$ Abnormal.

By R. S. Illingworth. 7th edn. Pp. xi +320 , illustrated. Churchill Livingstone, Edinburgh, London and New York, $\vec{P}$ 1980. $£ 10.00$

It is no surprise to find that this well known book has entere릉 its 7 th edition since it first appeared 20 years ago. The subjegt is one to which the author has himself made outstandify $Z$ personal contributions. The style is authoritative and charas teristically crisp and readable. The subject matter is concern with the assessment of both physical and mental developme巨t and it is supported by extensive data from the literature. The influence of prenatal and environmental factors are described and the methodology of assessment is given in some detai. $\vec{\varphi}$ Sub-sections are devoted to cerebral palsy and the evaluation of suitability for adoption. Throughout, the author is pains to indicate the limitations of the available techniques, the need for interpretation of the data obtained and the recognition of the errors which can occur. This is a book to be read and enjoyed by those learning about and working with $\bar{O}$ children. It is commended as an invaluable text for hospital and community doctors, and nurses involved in paediatric $\mathbb{Q}$ work and for the enquiring student.

\section{Diagnostic Electron Microscopy of Tumours}

By Feroze N. Ghadially. Pp. ix +251 , illustrated. Butterworths, London and Boston, 1980. £32.00.

The limitations of light microscopy in the diagnosis of $\vec{\partial}$ certain tumours are a recurrent source of frustration to 3 practising histopathologists and their clinical colleagues. Where available, ultrastructural examination can be of considerable help in identifying the nature of apparently anaplastic carcinomas, soft tissue sarcomas, and several other $\delta$ groups of tumours which share similar histological appearances. Professor Ghadially's new book aims to clarify this area of surgical pathology and to introduce the principles of tumour ultrastructure, mainly by dealing with a series of $I$ typical diagnostic problems.

After a brief introductory section on those aspects of $E$. M. technique relevant to diagnostic work, there is a section on $\widetilde{N}$ the ultrastructure of tumours in general and the differences between normal and neoplastic cells. Some basic knowledge of normal cellular structure is assumed in this book which can be regarded as complementary to the author's earlier volume Ultrastructural Pathology of the Cell. However, for those with such background knowledge, the current volume is self-contained.

\title{
,
}

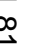

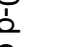


The main body of the book then addresses itself to answering specific diagnostic questions, with chapter titles such as 'Is it a carcinoma or a sarcoma?', 'Is it a melanoma?', 'Differential diagnosis of Ewing's tumour, neuroblastoma and lymphoma'. Other topics considered are apudomas, eosinophilic granular cell tumours, sarcomas and acute leukaemias: problems which are often unresolvable by light microscopy alone. For each problem the ultrastructural features that may be diagnostic are discussed in well documented detail, with critical assessment of published data where appropriate. Throughout, though, due caution is urged against overinterpretation of appearances, and clear criteria are given for diagnosis, notably in the chapter on myosarcomas (a discussion of intra-cytoplasmic filaments).

The last section describes a miscellany of rare tumours with distinctive ultrastructural appearances, and here the book becomes more of an atlas of tumour ultrastructure, reflecting the author's enthusiasm for his subject (few of us will ever see a juxtaglomerular cell tumour). Nonetheless, the electron micrographs here, as in the rest of the book, are of excellent quality, and go a long way towards justifying the high price.

Each chapter is written in a clear, sensible style, stressing the practical aspects of the subject, and is well referenced, with an emphasis on the author's own work. On the whole the topics considered are well covered, and there is sufficient detail for the histopathologists and pathology trainees for whom this book is intended, although more detailed accounts would have been useful for electron microscopists. However, the differential diagnosis of lymphomas could be considered in more detail-this subject is dismissed briefly, with little reference to the English literature. In the chapter on leukaemias, although the author has succumbed to the temptation to include rarities there is surprisingly no illustration of hairy cell leukaemia. A minor criticism is the rather large number of trivial misprints. Overall, though, the book is recommended as a useful one to have in any diagnostic surgical pathology laboratory with an electron microscope.

\section{Haemodynamic Basis of Atherosclerosis.}

By Meyer Tecon. Pp. xxi +95 , illustrated. Hemispherc Publishing Corporation, Washington, New York and London, 1980. £24.95.

The clinical problems resulting from atherosclerosis are of extreme major importance in clinical practice nowadays. This book presents a singular view point on the haemodynamic basis of atherosclerosis. Dr Meyer Texon takes a provocative stance on the subject regarding the aetiology of atherosclerosis, hypothesizing that the process is dependent on the laws of fluid dynamics rather than any abnormality of dietary intake or blood constituent. The author, in a clear manner of presentation, utilizing mathematical analyses and over 100 colour illustrations from his animal experiments and other research projects, develops his hypothesis that atherosclerosis is a haemodynamic phenomenon. The author develops his thesis in an extremely scientific fashion which is pleasing to the scientist in the field of fluid dynamics or to the specialist in cardiac disease and atherosclerosis, but is perhaps a little too complex for the general physician or student fully to understand or enjoy. There are adequate references at the end of each chapter with a full bibliography of the text and, if anything, the number of plates of his scientific experiments are perhaps a little unwieldy to the general discussion in the text. His basic thesis is that atherosclerosis may be considered a reactive biological process in the arteries to the forces generated by the blood flow. Therefore, atherosclerosis cannot be cured in the sense of curing an infectious disease or by alteration in dietary intake or a blood constituent and can only be controlled by a modification of blood velocity. This provocative view point has been well presented and should be essential reading to all those who are intimately involved in this major clinical problem. The basic fluid dynamics presented would be important reading for anybody involved in vascular surgery, and particularly coronary artery surgery, since the principles are most important in order to obtain satisfactory clinical results. This book is presented in an extremely interesting literary style with adequate illustrations to convey the more complex fluid dynamic principles and can be strongly recommended to all those who are involved in the problem of atherosclerosis.

\section{Health Service Counselling.}

By William Stewart. Pp. 175. Pitman Medical, London, 1979. $£ 4.95$ (paperback).

The author has addressed this book to those already employed in a counselling capacity within the health services and those who would like to incorporate counselling skills in their role in management, teaching or patient care. The simple and clear, if sometimes repetitious text invites the interested student to consider the principles of counselling and encourages him to seek some training in the field.

The opening section of this book deals with the process of counselling and its relevance to improving staff relationships. It examines ways of reducing personal and organizational stress through increased, effective communication. The author then tackles the difficult description of what constitutes counselling, and what does not.

The middle section considers an approach to the counselling interview and its exploration of problems. Finally, sample interviews and case studies are presented with an informative, analytical annotation.

This readable book would be well suited as an elementary introduction to the student of counselling.

\section{Lecture Notes on Gynaecology.}

By Josephine Barnes, 4th edn. Pp. 219, illustrated. Blackwell Scientific Publications, Oxford, 1980. $£ 4.75$ (paperback).

With an increased emphasis on Multiple Choice Questions, short and concise text books like Lecture Notes on Gynaecology have become increasingly popular, both in helping the student establish a framework in the subject and to assist in revision before examinations. The authoress, a Senior Consultant at Charing Cross Hospital and an Examiner for London and Oxford Universities has admirably succeeded in producing an up-to-date fourth edition of a very readable text book. This reviewer made a short list of topics that he felt should be covered in a new edition and found he had to cross them off one by one. The chapters on contraception and sterilization, and gynaecological endocrinology were full of fact and wise counsel. He would have liked more space devoted to therapeutic abortion, the management of the menopause and colposcopy.

The use of carbon dioxide for tubal patency and vaginal cytology for determination of hormonal status are no longer so relevant.

In a book as concise as this it would be worth-while havirg a list of general and specialist references at the end.

This book is very suitable for undergraduates taking their Final examinations and for nurses who wish to gain more medical insight into this speciality. Both will find the book accurate and enjoyable to read and marketed at a very reasonable price.

Lipids: A Clinician's Guide.

By L. A. Simons AND J. C. Gibson. Pp. $x+84$, illustrated. MTP Press, Lancaster, 1980. £7.50.

This book contains adequate information for the busy clinician or general practitioner who wishes to be better informed on the subject of lipids and lipoproteins with special reference 